\title{
Two-point correlation function in systems with van der Waals type interaction
}

\author{
Daniel Dantchev \\ Institut für Theoretische Physik, Technische Hochschule Aachen, 52056 Aachen, Germany \\ and \\ Institute of Mechanics, Bulgarian Academy of Sciences, Acad. G. Bonchev St. Building 4, 1113 Sofia, Bulgaria
}

(October 26, 2018)

\begin{abstract}
The behavior of the bulk two-point correlation function $G(\mathbf{r} ; T \mid d)$ in $d$-dimensional system with van der Waals type interactions is investigated and its consequences on the finite-size scaling properties of the susceptibility in such finite systems with periodic boundary conditions is discussed within mean-spherical model which is an example of Ornstein and Zernike type theory. The interaction is supposed to decay at large distances $r$ as $r^{-(d+\sigma)}$, with $2<d<4,2<\sigma<4$ and $d+\sigma \leq 6$. It is shown that $G(\mathbf{r} ; T \mid d)$ decays as $r^{-(d-2)}$ for $1 \ll r \ll \xi$, exponentially for $\xi \ll r \ll r^{*}$, where $r^{*}=(\sigma-2) \xi \ln \xi$, and again in a power law as $r^{-(d+\sigma)}$ for $r \gg r^{*}$. The analytical form of the leading-order scaling function of $G(\mathbf{r} ; T \mid d)$ in any of these regimes is derived.
\end{abstract}

64.60.-i, 64.60.Fr, 75.40.-s

\section{INTRODUCTION}

It is well known that the critical properties of a given statistical-mechanical system depend only on a small number of parameters like the dimensionality $d$ of the system, the symmetry of the order parameter characterizing the corresponding phase transition, and on general properties of the interaction coupling the order parameter at different locations. For example, in an isotropic $O(n)$ system one expects that all critical exponents and scaling function of a given physical quantity are independent on, say, lattice structure, or on short-range details of the interaction. Let us, for definiteness of notation, speak about Ising-like systems (i.e. $n=1$ ) with Hamiltonian

$$
\mathcal{H}=-\frac{1}{2} \sum_{\mathbf{r} \neq \mathbf{r}^{\prime}} J\left(\mathbf{r}-\mathbf{r}^{\prime}\right) S_{\mathbf{r}} S_{\mathbf{r}^{\prime}}
$$

In the context of the critical phenomena the usual criterion for a given interaction to be considered as short-ranged is finite second moment of $J(\mathbf{r})$, i.e. in terms of the Fourier transform $\tilde{J}(\mathbf{k})$ of this interaction for small $k=|\mathbf{k}|$ one has

$$
\tilde{J}(\mathbf{k})=\tilde{J}_{0}+\tilde{J}_{2} k^{2}+\Delta \tilde{J}(\mathbf{k}),
$$

where $\Delta \tilde{J}(\mathbf{k})$ is asymptotically smaller than $k^{2}$. Then, if $2<d<4$, for the bulk two-point correlation function

$$
G(\mathbf{r} ; T \mid d)=:<S_{\mathbf{r}} S_{\mathbf{0}}>-<S_{\mathbf{0}}>^{2}
$$

one normally writes

$$
G(\mathbf{r} ; T \mid d)=D r^{-(d-2+\eta)} X^{ \pm}(\mathbf{r} / \xi),
$$

where $X^{ \pm}$are two universal scaling functions (for $T>T_{c}$ and $T<T_{c}$, respectively), $D=D(T)$ is a nonuniversal, slowly varying function of temperature that well can be approximated by a constant in the vicinity of the critical point $T=T_{c}$ and $\xi$ is the bulk correlation length, i.e. $\xi(T) \simeq \xi_{0}^{+} t^{-\nu}, t \rightarrow 0^{+}$with $t=\left(T-T_{c}\right) / T_{c}$. For $T \geq T_{c}$ one has

$$
X^{+}(x) \simeq \begin{cases}\hat{X}^{+} x^{(d-3) / 2+\eta} \exp (-x), & x \rightarrow \infty \\ \text { constant, } & x \rightarrow 0 .\end{cases}
$$

The above is, in fact, the classical result of Fisher [1] for the two-point correlation function in the critical region of a simple fluid. Note, that when $r \gg \xi$ the correlations decay exponentially fast with the distance $r$. It is well known that in simple nonpolar fluids the interactions are characterized by potentials that decay as inverse powers of the distance at large $r$. In $d=3$ the most prominent case is the induced dipole-induced dipole (or van der Waals) interaction for which (neglecting the retardation effects) the potential decays as $\Phi(\mathbf{r}):=-J(\mathbf{r})=-A / r^{6}$, where $A>0$ is a positive 
constant. One easily can check that this interaction has a Fourier transform which is indeed of the type given in Eq. (1.2). But for such systems ( $d$-dimensional Ising model), in which the interactions decrease in inverse power with respect to the distance between the interacting objects, i.e. as $r^{-(d+\sigma)}, \sigma>2$, the following rigorous result due to Iagolnitzer and Souillard [2] is available.

Theorem [2]: The two-point correlation function $G(\mathbf{r} ; T, H \mid d)$ of a ferromagnetic system in the presence of an external magnetic field $H$ does not decay faster than its potential $J$. For any $T<\infty$ and any real $H$ there exists a strictly positive constant $C(T, H)$, such, that

$$
G(\mathbf{r} ; T, H \mid d)>C(T, H) J(\mathbf{r}) .
$$

For $H=0$ the theorem is valid for $T>T_{c}$ and for any of the two "pure" phases (the "plus" and the "minus" ones) for $T<T_{c}$.

The immediate consequence of this theorem is that if $T \neq T_{c}(1.4)$ - (1.5) could not be true for $r$ large enough independently on how close $T$ is to the critical point. The only way to reconcile (1.4) - (1.5) with the above theorem is to realise that if $T \neq T_{c}(1.4)-(1.5)$ could be valid only up to some $r=r^{*}(T)$. Then for $1 \ll r \ll \xi$ the correlations will decay as $r^{-(d-2+\eta)}$, for $\xi \ll r \ll r^{*}$ they will fall off exponentially, but, for $r>r^{*}$ they should again decay in a power law as a function of the distance, namely as $r^{-(d+\sigma)}$. In other words one should observe a crossover from power law to exponential and then, again, to power law behavior of the correlations. Saying this one immediately stacks with at least two important questions that appear naturally: 1) What is the value of $r^{*}$, i.e. where this crossover happens and 2) What are the properties of the function describing that crossover. One of the aims of the current article is to answer those questions in the framework of an exactly solvable model.

It is easy to check that one has the above situation only with interactions of the type $J(\mathbf{r}) \simeq A / r^{d+\sigma}$, where $\sigma>2$. To avoid misunderstanding in the remainder of the text let us make the following definitions. i) An interaction will be called of short range if for any finite $m$ its $m$-th moment is finite, i.e. $\sum_{\mathbf{r}} r^{m} J(\mathbf{r})<\infty$. ii) An interaction is long ranged if there exists a finite $m$ such that the corresponding $m$-th moment diverges. If $m=2$ this is a leading-order long range interaction, and if $m>2$ this is a subleading (van der Waals type) long-range interaction.

We recall that even if the interaction is short-ranged in the above sense (this is the situation we have with nearest neighbour, next-nearest neighbour, etc. interactions, i.e. with interactions that are essentially of a finite range), then (1.4) - (1.5) are again valid only for $1<<r<<r_{\mathrm{sr}}^{*}$. The exact results for $d=2$ Ising model (see, e.g. McCoy and $\mathrm{Wu}[3]$ ) and the mean-field results of Fisher and Burford [4] suggest that, if $T \neq T_{c}, r_{\mathrm{sr}}^{*} \sim \xi^{2}$. (The exact calculations due to Chen and Dohm [5] for the spherical model give a bit more "generous" estimation for $r_{\mathrm{sr}}^{*}$, namely $r_{\mathrm{sr}}^{*} \sim \xi^{3}$, see also below.) For $r>>r_{\mathrm{sr}}^{*}$ the interactions decay, of course, again exponentially, but they contain a nouniversal prefactor [5], i.e. their leading-order behavior is then nonuniversal. If the interaction is of a leading long-range type then (1.4) is also valid but in the limit $r / \xi \gg 1$ one has to require that $X^{+}(x) \simeq \hat{X}^{+} x^{\eta-2-\sigma}=\hat{X}^{+} x^{-2 \sigma}$, where we have taken into account that $\eta=2-\sigma$ if $\sigma<2[6] 8]$. The corrections to the large distance correlations in this case are in a power-law of $r$, which means that their leading order behavior is universal for any $r>>1$. This asymptotic is confirmed by the exact results for the spherical model due to Joyce [9,10] and it is in tune with the above theorem for the Ising model. Note that for $r$ large enough the correlations always fall off in a power law with the distance with the only exception of interactions of a fully finite range when they do decay exponentially.

That (1.4) - (1.5) should be modified for the case of subleading long-range interactions has been noticed by several authors.

First Widom proposed [1] that for $r \rightarrow \infty$

$$
G(\mathbf{r} ; T \mid d) \simeq \beta J(\mathbf{r})+a_{1} r^{-(d-1) / 2} \exp \left(-r / a_{2}\right),
$$

where $\beta=1 /\left(k_{B} T\right), k_{B}$ being the Boltzmann's constant, and $a_{1}$ and $a_{2}$ are "depending only on the thermodynamics state constants". It is clear that in nowadays formulations the above means to take for the correlation function a sum of $\beta J(\mathbf{r})$ and the right-hand side of Eq. (1.4). Later the problem has been attacked by Enderby, Gaskell and March [12]. They consider a three-dimensional fluid, i.e. the case $d=\sigma=3$. Supposing the Ornstein-Zernike integral equation to be valid and taking the direct correlation function to be $c(r)=\beta J(r)$, they obtain, after assuming that the structure factor $S$ has a Fourier transform of the type $S(k)=\chi / \beta+c_{2} k^{2}+c_{3} k^{3}+\cdots$, that $G(\mathbf{r} ; T \mid 3) \simeq J(r) \chi^{2} / \beta=A \chi^{2} /\left(\beta r^{6}\right)$, when $r \rightarrow \infty$ and in temperature regions "well away" from the critical point [13. Starting from this result, Kayser and Raveché 14 suggest that $G(\mathbf{r} ; T \mid d)$ can be decomposed in two additive contributions $G^{\mathrm{sr}}$ and $G^{\mathrm{lr}}$, where $G^{\mathrm{sr}}$ is given by (1.4), plus higher-order terms that account for the usual corrections to scaling, and $G^{\mathrm{lr}}=\Theta\left(r-r^{*}\right) J(r) \chi^{2} / \beta$. Here $\Theta(x)$ is the Heaviside step function and $r^{*}$ is to be determined by the requirement that at this point $G^{\mathrm{sr}}=G^{\mathrm{lr}}$. Taking $\chi \propto \xi^{2-\eta}$ in the expression for $G^{\mathrm{lr}}$, which in fact means supposing $G(\mathbf{r} ; T \mid d) \simeq J(r) \chi^{2} / \beta, r \rightarrow \infty$, to be valid for general $d$ and also for temperatures close to $T_{c}$, the above authors derived

$$
r^{*}=(\sigma-2+\eta) \xi \ln \xi
$$


In 15] Flöter and Dietrich make similar statements for $r^{*}$ [16] for the case $d=\sigma=3$.

In the present article we will investigate the large $r$ behavior of the correlations and will derive the explicit form of $G^{\mathrm{lr}}$ within the mean spherical model. The interaction will be supposed to be of the type $J=A / r^{d+\sigma}$, with $2<d<4$, $2<\sigma<4$ and $d+\sigma \leq 6$.

If one knows $G$ one can immediately determine the behavior of the bulk susceptibility $\chi$ by using the fluctuationdissipation relationship $\chi(T \mid d)=\beta \sum_{\mathbf{r}} G(\mathbf{r} ; T \mid d)$. Definitely, if the finite-size two-point correlation function is known for a given finite system with a characteristic size $L$, then one can determine in this way also the behavior of the finite-size susceptibility $\chi(T ; L \mid d)$. In a recent article [5] Chen and Dohm have addressed the question: could one say what should be the scaling structure of the finite-size susceptibility under periodic boundary conditions if one knows only the bulk two-point correlation function? They suggest a hypothesis, that this is possible by interpreting in a proper way the functional dependence of $G$ on $r$ as a dependence of $\chi(T ; L \mid d)$ on $L$. In the present article we check the relationship that they suggest between $G$ and $\chi(T ; L \mid d)$ on the example of our exactly solvable model. For that aim we will use the results for $\chi(T ; L \mid d)$ derived in [17] for the same model.

The structure of the article is as follows. In Section II we briefly describe the model and present our starting analytical expressions. Section III contains our results for the large $r$ behavior of the two-point correlation function $G$. In Appendix A we present some details of the calculations needed to determine the asymptotics of $G$ as a function of $r$ and $\xi$. Section IV comments on the relationship between the derived results for $G$ and the behavior of the finitesize susceptibility of systems with subleading long-range interactions. The article closes with a Discussion (Section V) where we speculate about the possible extensions of our results for other models.

\section{THE MODEL}

We consider a $d$-dimensional mean spherical model [18], [19] (for a comprehensive review on the results available for this model see [20]). The degrees of freedom consist of a set of $N$ localised spins with Gaussian weight, and the Hamiltonian is given by Eq. (1.1). The interaction $J(\mathbf{r})$ is supposed to be of van der Waals type, i.e. its Fourier transform is supposed to be of the form

$$
\tilde{J}(\mathbf{k}) \simeq \tilde{J}(\mathbf{0})\left(1-v_{2} k^{2}+v_{\sigma} k^{\sigma}-v_{4} k^{4}+O\left(k^{6}\right)\right),
$$

where $k=|\mathbf{k}|, 4>\sigma>2$ and $\tilde{J}(\mathbf{0}), v_{2}, v_{\sigma}$ and $v_{4}$ are nonuniversal positive constants. Note that the signs of the coefficients in the small $k$ expansion of the Fourier transform of the interaction are chosen so as they normally appear for subleading long-range interactions that decay in power law with the distance between the interacting objects - molecules or spins. In (2.1) $\tilde{J}(\mathbf{0}), v_{2}, v_{\sigma}$ and $v_{4}$ are $\sigma$-dependent - for simplicity of notation this dependence is omitted here. The term $v_{\sigma} q^{\sigma}$ in (2.1) is associated with a contribution to the real-space interaction going as $r^{-d-\sigma}$. Furthermore, we suppose that $\tilde{J}(\mathbf{k})-\tilde{J}(\mathbf{0})<0$ if $\mathbf{k} \neq \mathbf{0}$, which reflects the fact that there are no competing interactions in the system and that the only ground state is the ferromagnetic one. Of course, it would be interesting to consider such systems - say with a combination between antiferromagnetic short range and ferromagnetic subleading long-range interactions, but this is out of the scope of the current article.

The partition function of the model is given by the multiple integral

$$
\int_{-\infty}^{\infty} d s_{1} \cdots \int_{-\infty}^{\infty} d s_{N} \exp [-\beta \mathcal{H}]
$$

supplemented by the mean spherical condition

$$
\sum_{i=1}^{N}\left\langle s_{i}^{2}\right\rangle=N,
$$

which can be enforced with the use of a "Lagrange multiplier" term going as $\lambda \sum_{i=1}^{N} s_{i}^{2}$ into the effective Hamiltonian, and thence into the partition function. The spherical model equation of state then takes the form

$$
\sum_{\mathbf{k}} \frac{k_{B} T}{\lambda-\tilde{J}(\mathbf{0})\left(1-v_{2} k^{2}+v_{\sigma} k^{\sigma}-v_{4} k^{4}\right)}=N
$$

The phase transition in this model occurs when the combination $\lambda-\tilde{J}$ takes on a value asymptotically close to zero. The difference between the equation of state in (2.4) and the standard mean spherical model condition in short range 
systems lies in the addition of the term going as $k^{\sigma}$ in the denominator on the left hand side of (2.4). In general, this term is taken to be negligible, but we will soon see that it leads to interesting effects.

For the model defined in the above way it can be shown, following [10], that the bulk correlation function $G(\mathbf{r} ; K \mid d, \sigma)$ is given by, if $2<d<4$,

$$
G(\mathbf{r} ; K \mid d, \sigma)=\frac{1}{K} \frac{1}{(2 \pi)^{d}} \int_{\mathcal{R}^{d}} \frac{e^{i \mathbf{k} \cdot \mathbf{r}} d \mathbf{r}}{\xi^{-2}+k^{2}-b k^{\sigma}+c k^{4}},
$$

where $K=\beta v_{2} \tilde{J}(\mathbf{0})$, and $b=v_{\sigma} / v_{2}>0$ and $c=v_{4} / v_{2}>0$ are nonuniversal constants. Let us note that the values of $b$ and $c$ are such, that there are no real roots of the equation $1-b k^{\sigma-2}+c k^{2}=0$. The last follows from the propositions we made for $\tilde{J}(\mathbf{k})$. Note also that in $(2.5)$ we have taken the cut-off in the $k$-space to be infinity (for a lattice system it will mean that one considers the limit of a zero lattice spacing). This is possible because of the rapid oscillations of the exponential function in the integrand, but in this way we neglect all finite cut-off effects that will give nonuniversal contributions towards the critical behavior of the two-point correlation function (see [5] for details). In 2.5) $\xi=\xi_{2}$ is the second moment correlation length defined via (see, e.g. [21])

$$
\xi^{2}=-\left.[\tilde{G}(\mathbf{0} ; K \mid d, \sigma)]^{-1} \frac{\partial}{\partial k^{2}} \tilde{G}(\mathbf{k} ; K \mid d, \sigma)\right|_{k=0}
$$

where $\tilde{G}(\mathbf{k} ; K \mid d, \sigma)$ is the Fourier transform of $G(\mathbf{r} ; K \mid d, \sigma)$. Because of this identification one can, in fact, skip for our purposes the analysis of the spherical field equation (2.4) - one directly has $\lambda=\tilde{J}(\mathbf{0})\left(1+v_{2} \xi^{-2}\right)$.

Since $\sigma>2$ and since we are interested in the behavior of $G(\mathbf{r} ; K \mid d, \sigma)$ for $|\mathbf{r}| \gg 1$ (note that then the leading order contributions of the integral in (2.5) will be coming from small $k$ values), one can rewrite (2.5) in the form

$$
G(r ; K \mid d, \sigma)=G^{\mathrm{sr}}(r ; K \mid d)+G^{\mathrm{lr}}(r ; K \mid d, \sigma),
$$

i.e. as a sum of "short-range" and "long-range" parts. The "short-range" correlation function is the part that is only due to the short-range component of the interaction and, as usual, will be taken to be of the form

$$
G^{\mathrm{sr}}(r ; K \mid d)=\frac{1}{K} \frac{1}{(2 \pi)^{d}} \int_{\mathcal{R}^{d}} \frac{e^{i \mathbf{k} \cdot \mathbf{r}} d \mathbf{r}}{\xi^{-2}+k^{2}} .
$$

The other contributions that are due to the subleading components of the interaction do form the corresponding "long-range" part. As it has been already stated in Section 1 such a structure has been supposed to hold by Kayser and Ravechè [14] in their qualitative analysis of the correlation functions in fluids.

Performing the integrations in (2.8) and taking into account that for $2<\sigma<4$ and $r \gg 1$

$$
\begin{aligned}
G(r ; K \mid d, \sigma) & =G^{\mathrm{sr}}(r ; K \mid d)+\frac{1}{K} \frac{1}{(2 \pi)^{d}} \int_{\mathcal{R}^{d}} \frac{\left(b k^{\sigma}-c k^{4}\right) e^{i \mathbf{k} \cdot \mathbf{r}} d \mathbf{r}}{\left(\xi^{-2}+k^{2}-b k^{\sigma}+c k^{4}\right)\left(\xi^{-2}+k^{2}\right)} \\
& \simeq G^{\mathrm{sr}}(r ; K \mid d)+\frac{b}{K} \frac{1}{(2 \pi)^{d}} \int_{\mathcal{R}^{d}} \frac{k^{\sigma} e^{i \mathbf{k} \cdot \mathbf{r}} d \mathbf{r}}{\left(\xi^{-2}+k^{2}\right)^{2}}+\cdots,
\end{aligned}
$$

we obtain

$$
G(r ; K \mid d, \sigma)=\frac{1}{K} \frac{1}{(2 \pi)^{d / 2}} r^{-(d-2)}\left[X^{\mathrm{sr}}(r / \xi)+b r^{-(\sigma-2)} X^{\mathrm{lr}}(r / \xi)+\cdots\right]
$$

where

$$
\begin{gathered}
X^{\mathrm{sr}}(x)=x^{(d-2) / 2} K_{(d-2) / 2}(x), \\
X^{\operatorname{lr}}(x)=\frac{\pi}{\sin [(d+\sigma) \pi / 2]}\left\{2^{d / 2+\sigma-4}{ }_{1} \tilde{F}_{2}\left(2 ; 2-\sigma / 2,3-d / 2-\sigma / 2 ; \frac{x^{2}}{4}\right)\right. \\
\left.-\frac{1}{4} x^{d / 2+\sigma-3}\left[x I_{d / 2}(x)-(d+\sigma-2) I_{d / 2-1}(x)\right]\right\},
\end{gathered}
$$

and $\cdots$ stays for contributions which are corrections with respect to the terms retained. Here $I_{a}(x)$ is the modified Bessel function, and ${ }_{p} \tilde{F}_{q}(\mathbf{a} ; \mathbf{b} ; z)$ is the regularized generalized hypergeometric function 


$$
{ }_{p} \tilde{F}_{q}(\mathbf{a} ; \mathbf{b} ; z)=\frac{{ }_{p} F_{q}(\mathbf{a} ; \mathbf{b} ; z)}{\Gamma\left(b_{1}\right) \Gamma\left(b_{2}\right) \cdots \Gamma\left(b_{q}\right)},
$$

where ${ }_{p} F_{q}(\mathbf{a} ; \mathbf{b} ; z)$ is the generalized hypergeometric function

$$
{ }_{p} F_{q}(\mathbf{a} ; \mathbf{b} ; z)=\sum_{k=0}^{\infty} \frac{\left(a_{1}\right)_{k}\left(a_{2}\right)_{k} \cdots\left(a_{p}\right)_{k}}{\left(b_{1}\right)_{k}\left(b_{2}\right)_{k} \cdots\left(b_{q}\right)_{k}} \frac{z^{k}}{k !}
$$

The symbol $(a)_{k}=a(a+1) \cdots(a+k-1)=\Gamma(a+k) / \Gamma(a)$ in the above equation is the Pochhammer's symbol. The function ${ }_{p} \tilde{F}_{q}$ is finite for all finite values of its arguments. In the above expressions only the leading order long-range contributions (i.e. the contributions "proportional to $b$ "), have been retained and we have supposed that $2<d<4$, $2<\sigma<4$, and $d+\sigma<6$.

We recall that for the Ornstein-Zernike type theories (including the mean-spherical model, see, e.g. [10]) $\eta=0$. In Section $\mathrm{V}$ we will discuss briefly the generalization of (2.10) for models with $\eta \neq 0$.

The expressions (2.10)-(2.12) are the analytical basis for our further analysis. Let us note that the correlations within the spherical model have been a subject of detailed investigations (see [10] and [20] for a comprehensive review) for both short-range and leading long-range interactions. Surprisingly enough, they have never been investigated for subleading long-range interactions.

\section{LARGE DISTANCE BEHAVIOR OF THE BULK TWO-POINT CORRELATION FUNCTION}

The asymptotics of the scaling function $X^{\text {sr }}$ for $2<d<4$ are well known (see, e.g. [23] and references cited therein)

$$
X^{\mathrm{sr}}(x) \simeq \begin{cases}\sqrt{\frac{\pi}{2}} x^{(d-3) / 2} \exp (-x)\left(1+O\left(x^{-1}\right)\right), & x \rightarrow \infty \\ \frac{\Gamma(d / 2-1)}{2^{(4-d) / 2}}+\frac{\pi x^{d-2}}{2^{d / 2} \sin (\pi d / 2) \Gamma(d / 2)}+O\left(x^{2}\right), & x \rightarrow 0 .\end{cases}
$$

Let us, nevertheless, make some comments here. First, let us note that the above asymptotic is obtained if one makes a quadratic approximation of the spectrum and lets the cut-off $\Lambda$ of the theory go to infinity. (If one keeps a sharp finite cut-off $\Lambda$ with such an approximation of the spectrum one will obtain a nonexponential oscillatory power-law behavior [5], 221). Second, on a lattice, for nearest neighbours interactions between the spins embedded in a $d$-dimensional cube it has been shown that the above expression is valid [0] only for $1<<r<<\xi^{3}$. If $r \geq \xi^{3}$ the correlations do depend (up to the leading order) on the mutual positions of the spins involved, i.e. the lattice anisotropy comes into the play and can no longer be neglected [5]. So, one can think that the above expression is valid in the region $1<<x<<\xi^{2}$. Third, for $x \rightarrow 0$ the second term in this short-range expansion of the correlation function involves a $t$ dependence of the type $t^{\alpha}$ if one takes into account that $\xi \simeq \xi_{0}^{+} t^{-\nu}$ for $t \rightarrow 0^{+}$and that $(d-2) \nu=\alpha$, i.e.

$$
X^{\mathrm{sr}}(r / \xi) \simeq \frac{\Gamma(d / 2-1)}{2^{(4-d) / 2}}+\frac{\pi\left(r / \xi_{0}^{+}\right)^{d-2}}{2^{d / 2} \sin (\pi d / 2) \Gamma(d / 2)} t^{\alpha}+O\left((r / \xi)^{2}\right), \xi \gg r .
$$

The temperature dependent term in this expansion is usually not explicitly specified in the literature on the spherical model. Finally, let us note that according to the above asymptotics and under the approximations made $\xi_{2}=\xi_{e}=\xi$, i.e. the second-moment correlation length coincides in such a theory with the exponential-decay correlation length.

(In [0] it has been shown that for a model on a hypercubic lattice $\xi_{2}=a /\left[2 \sinh \left(a / 2 \xi_{e}\right)\right]$, where $a$ is the lattice spacing and $\xi_{e}$ has been chosen to be along one of the principal axis of the lattice.)

The asymptotics of $X^{\mathrm{lr}}$ for $2<d<4,2<\sigma<4, d+\sigma<6$ are (see Appendix A for a derivation)

$$
X^{\operatorname{lr}}(x) \simeq \begin{cases}-2^{\sigma+d / 2-2} \frac{\sigma(d+\sigma-2) \Gamma((d+\sigma) / 2-1)}{\Gamma(1-\sigma / 2)} x^{-4}+O\left(x^{-6}\right), & x \rightarrow \infty \\ 2^{\sigma+d / 2-4} \frac{\Gamma((d+\sigma) / 2-2)}{\Gamma(2-\sigma / 2)}-x^{d+\sigma-4} \frac{\pi(d / 2+\sigma / 2+1)}{2^{d / 2} \Gamma(d / 2) \sin (\pi(d+\sigma) / 2)}+O\left(x^{2}\right), & x \rightarrow 0 .\end{cases}
$$

The above asymptotics lead to the following behavior of the short and long-range parts of the bulk two-point correlation function

$$
G^{\mathrm{sr}}(r ; K \mid d) \simeq \frac{1}{K} \frac{\sqrt{\pi / 2}}{(2 \pi)^{d / 2}} \xi^{-(d-3) / 2} r^{-(d-1) / 2} \exp (-r / \xi)\left(1+O\left((r / \xi)^{-1}\right)\right), r \gg \xi,
$$


and

$$
G^{\mathrm{l}}(r ; K \mid d, \sigma) \simeq-\frac{b}{K} \frac{2^{\sigma-2}}{\pi^{d / 2}} \sigma(d+\sigma-2) \frac{\Gamma((d+\sigma) / 2-1)}{\Gamma(1-\sigma / 2)} \xi^{4} r^{-(d+\sigma)}, r \gg \xi
$$

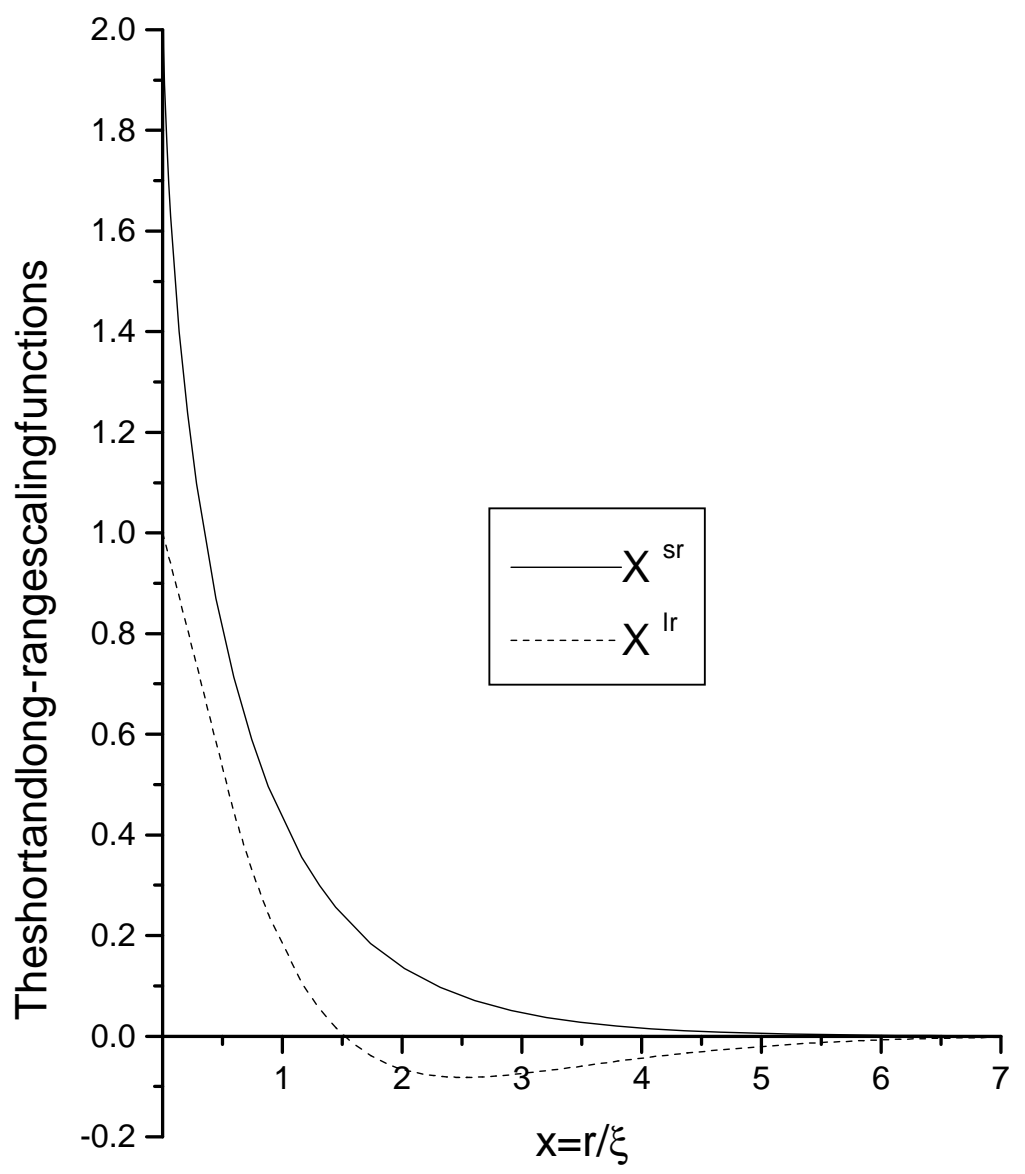

FIG. 1. We present as illustration plots of the scaling functions $X^{\mathrm{sr}}(x)$ and $X^{\mathrm{lr}}(x)$ of the short-range and long-range correlation functions, respectively, as a function of the scaling variable $x=r / \xi . X^{\mathrm{sr}}(x)$ is plotted for $d=2.5$, whereas $X^{\mathrm{lr}}(x)$ is for $d=3$ and $\sigma=2.5$. For any $2<d<4, X^{\text {sr }}$ is always positive and decays monotonically as a function of $x$. For large values of $\mathrm{x}, X^{\mathrm{sr}}$ decays exponentially fast. Note that in contrast with $X^{\mathrm{sr}}(x), X^{\mathrm{lr}}(x)$ is not a monotonic function of $x$. In addition $X^{\mathrm{lr}}$ can be both positive and negative. $X^{\mathrm{lr}}(x)$ decays in a power law, as $x^{-4}$, for large values of its argument.

One can determine the crossover region where the correlations from short range become long range type. To that aim one has to solve the equation

$$
G^{\mathrm{sr}}(r ; K \mid d) \simeq G^{\mathrm{lr}}(r ; K \mid d, \sigma) .
$$

Having in mind Eqs. (2.10), (3.4) and (3.5) one obtains that the crossover takes place at $r \simeq r^{*}$, where

$$
r^{*}=(\sigma-2) \xi \ln \xi+\left(\frac{d+1}{2}+\sigma\right) \ln \ln \xi
$$

The leading-order term of this result coincides with that one given in [14] (if one takes into account that $\eta=0$ for the model under consideration).

For $d=\sigma=3$, i.e. for the true van der Waals interaction, the corresponding scaling functions are

$$
X^{\mathrm{sr}}(x)=\sqrt{\frac{\pi}{2}} \exp (-x)
$$

and 


$$
X^{\operatorname{lr}}(x)=\sqrt{\frac{2}{\pi}}\left\{1-\frac{3}{4} x[\exp (-x) \operatorname{Ei}(x)-\exp (x) \operatorname{Ei}(-x)]+\frac{1}{4} x^{2}[\exp (-x) \operatorname{Ei}(x)+\exp (x) \operatorname{Ei}(-x)]\right\} .
$$

The asymptotics of the short-range scaling function are obvious, while these for the long-range one are

$$
X^{\operatorname{lr}}(x) \simeq \begin{cases}24 \sqrt{2 / \pi} x^{-4}\left(1+O\left(x^{-6}\right)\right), & x \rightarrow \infty \\ \sqrt{2 / \pi}\left(1+2 x^{2} \ln x\right)+O\left(x^{2}\right), & x \rightarrow 0 .\end{cases}
$$

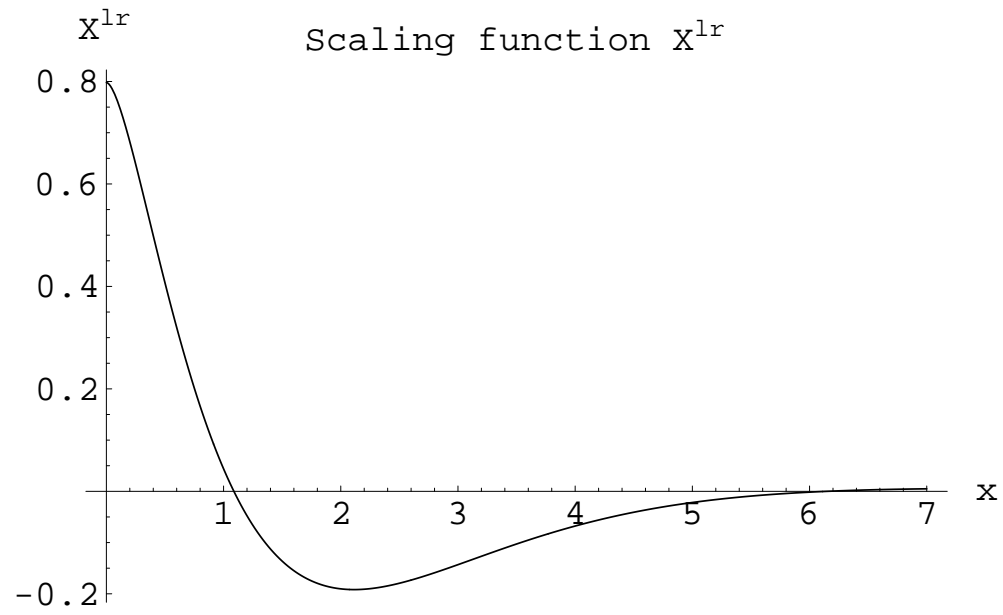

FIG. 2. The scaling function $X^{\mathrm{lr}}(x)$ of the long-range correlation function for $d=\sigma=3$. One observes that, in contrast with the short-range correlation function, it is not a monotonic function of the scaling variable $x=r / \xi$. It changes sign at $x \simeq 1.088$ and $x \simeq 6.146$ and reaches a minimum at $x_{\min } \simeq 2.113$ which is $X^{\mathrm{lr}}\left(x_{\min }\right) \simeq-0.192$. In other words the long range part of the interaction increases the correlations (in comparison with an effective short-range system having the same value of $K$; we recall that $K$ is a $\sigma$-dependent quantity) for $r$ up to $1.088 \xi$ and for $r>6.145 \xi$, but decreases them for $1.088 \xi<r<6.146 \xi$. The maximum of $X^{\mathrm{lr}}$ is reached at $x=0$ and it is $X^{\operatorname{lr}}(0)=\sqrt{2 / \pi} \simeq 0.798$. The last implies, as it is to be expected, that for a fixed $r$ the maximal increment of the correlations due to the long-range part of the interaction is reached at $T=T_{c}$. $X^{\mathrm{lr}}(x)$ decays in a power law, as $x^{-4}$, for large values of its argument.

The asymptotics of the correlation function at $T=T_{c}$ and for any fixed $\xi$ can be derived to much greater details in the limit $r \rightarrow \infty$ for this especially important case. They are (see Appendix A)

$$
G(\mathbf{r} ; K \mid 3,3)=\frac{b}{K} \frac{12}{\pi^{2}} \frac{\xi^{4}}{r^{6}}\left[1+120(r / \xi)^{-2}+10080\left(1-\frac{2}{3} \frac{c}{\xi^{2}}\right)(r / \xi)^{-4}+O\left((r / \xi)^{-6}\right)\right], r \gg \xi,
$$

and

$$
G\left(\mathbf{r} ; K_{c} \mid 3,3\right)=\frac{1}{K_{c}} \frac{1}{4 \pi r}\left[1+\frac{2 b}{\pi} r^{-1}-\frac{4}{\pi} b\left(b^{2}-2 c\right) r^{-3}+O\left(r^{-5}\right)\right], r \rightarrow \infty .
$$

One can easily check that up to the leading-order terms these asymptotics coincide with the corresponding ones that follow by using the behavior of the short- and long-range correlation functions given above. For example, when $r \rightarrow \infty$ but $r \ll \xi$ (i.e. $x=r / \xi \rightarrow 0$ ) from Eqs. (3.8) and (3.9) one has

$$
G(\mathbf{r} ; K \mid 3,3)=\frac{1}{K} \frac{1}{4 \pi r}\left[1+x+\frac{1}{2} x^{2}+\frac{2 b}{\pi} r^{-1}-\frac{4 b}{\pi} r^{-1} x^{2} \ln x+O\left(r^{-3}, x^{3}, x^{2} r^{-1}\right)\right], r \rightarrow \infty, x=r / \xi \rightarrow 0 .
$$

The crossover from short-range to long-range type behavior happens at $r=r^{*}$ where $r^{*}$, in full agreement with Eq. (3.7) for $d=\sigma=3$, is given by

$$
r^{*}=\xi\left\{\ln \xi+5 \ln \ln \xi+O\left(\frac{\ln \ln \xi}{\ln \xi}\right)\right\}
$$


Note that Eq. (3.7) was derived under the condition that $d+\sigma<6$ and its "analytical continuation" to $d=\sigma=6$ is not obvious. It is nevertheless valid because for $r>>\xi$ the leading-order term in the behavior of $G(\mathbf{r} ; K \mid 3,3)$ (see Eq. (3.11)) can be obtained from that one of $G(\mathbf{r} ; K \mid d, \sigma)$, given by Eq. (3.5), if one sets $d=\sigma=3$ there.

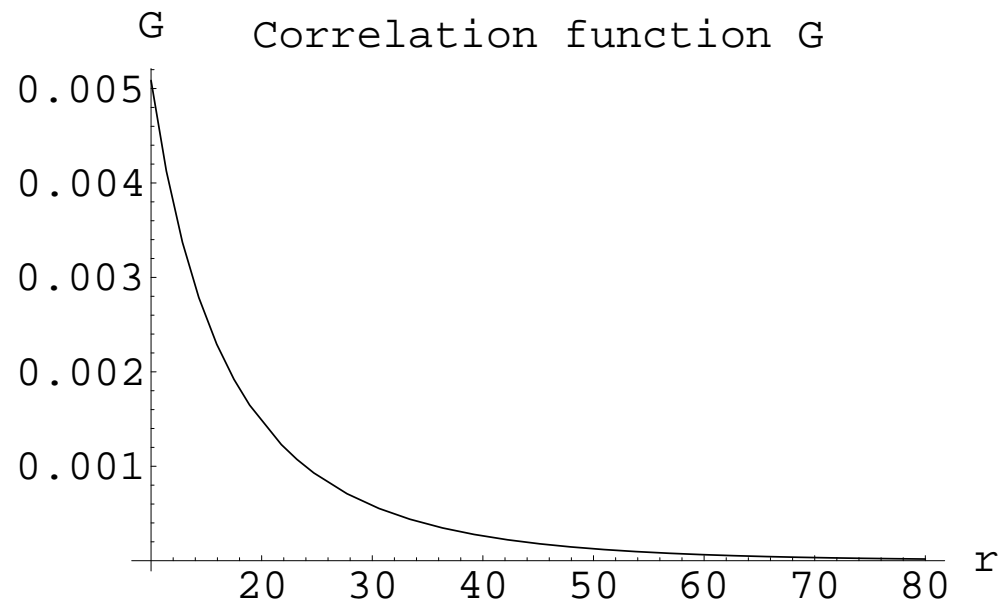

FIG. 3. We give here a representative example of the total two-point correlation function $G(\mathbf{r} ; K \mid d, \sigma)$ as a function of $r$ for $d=\sigma=3$ and $K=b=1, \xi=20$. As it should be expected for a ferromagnetic system $G>0$ and decays monotonically as a function of $r$.

\section{FINITE-SIZE SCALING SUSCEPTIBILITY}

One usually describes the critical behavior of finite systems in the framework of the finite-size scaling theory 20,24 27. The standard finite-size scaling is usually formulated in terms of only one reference length, namely the bulk correlation length $\xi$. The main statements of the theory are that

i) The only relevant variable in terms of which the properties of the finite system depend in the neighbourhood of the bulk critical temperature $T_{c}$ is $L / \xi$.

ii) The rounding of the phase transition in a given finite system sets in when $L / \xi=O(1)$.

The tacit assumption is that all other reference lengths (such as lattice spacings, inverse cut-off, etc.) will lead only to corrections in the above picture. In addition, by analogy with the bulk short-range systems it is supposed that if $\sigma \geq 2$ the finite-size critical behavior will be that of the corresponding short-ranged finite-size systems (see, e.g. [28]), characterised by exponentially fast decay of the finite-size dependence of the thermodynamic quantities at least when the critical region of the system is leaved in the direction towards higher temperatures (the low-temperature behavior depends on additional features like existence, or not, of spin-wave excitations - Goldstone bosons).

As it has been recently shown the above picture is, in fact, more complicated [5],29] and not completely valid for systems with subleading long-range interactions 17. For such systems within the mean-spherical model and under periodic boundary conditions it has been found that the finite-size susceptibility $\chi(t ; L)$ is of the form [17]

$$
\chi(t ; L)=L^{\gamma / \nu} Y\left(x_{1}, b L^{2-\sigma}\right),
$$

or, equivalently,

$$
\chi(t ; L)=L^{\gamma / \nu}\left[Y^{\mathrm{sr}}\left(x_{1}\right)+b L^{2-\sigma} Y^{\mathrm{lr}}\left(x_{1}\right)\right],
$$

where $x_{1}=a t L^{1 / \nu}$, and $Y, Y^{\mathrm{sr}}$ and $Y^{\mathrm{lr}}$ are universal functions. The quantities $a$, and $b$ are nonuniversal constants. One quite common way of fixing $a$ is to choose it to be $a=\left(\xi_{0}^{+}\right)^{-1 / \nu}$. It is worthily to note the close similarity in the structures of Eqs. (2.10) and (4.2). In other words - if one knows the structure of the bulk two-point correlation function one easily can write the corresponding finite-size behavior of the susceptibility. A hypothesis about such a possibility has been stated for the first time in $[5]$.

In the high-temperature, disordered phase, where $t L^{1 / \nu} \rightarrow \infty$, we find that the long-range portion of the spin-spin interaction gives rise to contributions of the order of $b L^{-(d+\sigma)}$ that swamp the exponentially small terms that are expected to characterise the signature of finite size in systems with periodic boundary conditions and short range interactions. In other words the subleading long-range part of the interaction gives rise to a dominant finite-size 
dependence in this regime. This is entirely consistent with the inherent long-range correlations that accompany long-range interactions, but it violates the standard finite-size scaling formulation. More explicitly, one obtains $Y^{\mathrm{sr}}\left(x_{1}\right) \sim \exp \left(-\right.$ const. $\left.x_{1}^{\nu}\right)$, while

$$
Y^{\operatorname{lr}}\left(x_{1}\right) \sim x_{1}^{-d \nu-2 \gamma}
$$

when $x_{1} \rightarrow \infty$. This asymptotic follows from the requirement the finite-size corrections to be of the order of $L^{-(d+\sigma)}$ in this regime, which is to be expected on general grounds and is supported by the existing both exact and perturbative results for models with leading long-range interaction included [30 32]. Note that (4.3) implies for the temperature dependence of this correction

$$
\chi(t ; L)-\chi(t ; \infty) \sim t^{-d \nu-2 \gamma} L^{-(d+\sigma)}, t L^{1 / \nu} \rightarrow \infty
$$

Obviously, the existence of such power-law finite-size dependent dominant terms above $T_{c}$ is of significance in the analysis of Monte Carlo data for such systems.

Let us now consider the case $d+\sigma=6,2<d<4,2<\sigma<4$, which contains the genuine van der Waals interaction with $d=\sigma=3$. Then, instead of (4.2), one has 17

$$
\chi(t ; L)=L^{\gamma / \nu}\left\{Y^{\mathrm{sr}}\left(x_{1}\right)+b L^{2-\sigma}\left[Y_{1}^{\operatorname{lr}}\left(x_{1}\right) \ln (L)+Y_{2}^{\operatorname{lr}}\left(x_{1}\right)\right]\right\} .
$$

Comparing with the corresponding results for the correlation function one observes, since there is no explicit ln $r$ dependence there, that this subtle feature like the logarithmic-in- $L$ corrections will not be captured in the above mentioned approach — from the structure of the bulk two-point correlation function with respect to $r$ to obtain that one of the finite size susceptibility with respect to $L$ (simply by considering $L$ in the role of $r$ ). Nevertheless, indications that the situation here may be more complicated are found in the short distance expansion of the bulk correlation function which has logarithmic in $r$ terms.

\section{CONCLUDING REMARKS AND DISCUSSION}

In the present article we derived the analytical behavior of the two-point correlation function in a system with van der Waals type interaction. The treatment has been made within the mean-spherical model, which is an example of Ornstein and Zernike type theory. We have pointed out that the leading order behavior of $G(\mathbf{r} ; T \mid d)$ as a function of the distance is exponential only within the region of separations $r$ between the interacting objects given by the condition $\xi \ll r \ll r^{*} \equiv(\sigma-2) \xi \ln \xi$. Obviously, taking into account the dependence of $\xi$ on the temperature, this region widens essentially only very close to $T=T_{c}$. When $r$ is outside the region defined above the correlations decay in a power law as a function of $r$ : as $r^{-(d-2)}$ for $r \ll \xi$, and as $r^{-(d+\sigma)}$ for $r \gg(\sigma-2) \xi \ln \xi$. It turns out that $G(\mathbf{r} ; T \mid d)$ can be decomposed in a "short-range" and "long-range" parts (see Eq. (2.7)). The corresponding shortand long-range scaling functions $X^{\mathrm{sr}}$ and $X^{\mathrm{lr}}$ are given in Eqs. (2.11) and (2.12), respectively. For the case $2<d<4$, $2<\sigma<4$ and $d+\sigma<6$ the behavior of these functions is illustrated in Fig. 1, whereas the small and large value asymptotics of the functions are given in Eqs. (3.1) and (3.3). A special attention is paid to the most important case of $d=\sigma=3$ which mimics the real van der Waals interaction in fluids. The analytical expressions for the scaling functions are given in Eqs. (3.8) and (3.9). The behavior of $X^{\mathrm{lr}}$ is plotted on Fig. 2. The asymptotics of $G(\mathbf{r} ; K \mid 3,3)$ and $G\left(\mathbf{r} ; K_{c} \mid 3,3\right)$ are given in Eqs. (3.11) and (3.12). The behavior of the total correlation function $G(\mathbf{r} ; K \mid 3,3)$ is illustrated in Fig. 3 .

Let us note that since all of the above results are for the mean spherical model they pertain to the case of $\eta=0$ models. Naturally, one stacks with the question: How expressions like (2.10) and (3.7) should be modified for models with $\eta \neq 0$ ? A hint in this direction can be found in [14 - for such models Kayser and Raveché suggest that, in our terminology, $r^{*}=(\sigma-2+\eta) \xi \ln \xi$. In order to reconcile this statement with Eq. (2.10) one has to suppose that when $\eta \neq 0$ (see also Eq. (1.4))

$$
G(r ; K \mid d, \sigma)=D(T) r^{-(d-2+\eta)}\left[X^{ \pm, \mathrm{sr}}(r / \xi)+b r^{-(\sigma-2+\eta)} X^{ \pm, \operatorname{lr}}(r / \xi)+\cdots\right] .
$$

Here $X^{\mathrm{sr}}$ is supposed to have the usual properties (see Eq. (1.5)), whereas for $X^{\mathrm{lr}}$ we suppose that $X^{\mathrm{lr}}(x) \rightarrow X_{-}^{\mathrm{lr}}, x \rightarrow 0$ and $X^{\mathrm{lr}}(x) \rightarrow X_{+}^{\mathrm{lr}} x^{-2(2-\eta)}, x \rightarrow \infty$, where $X_{-}^{\mathrm{lr}}$ and $X_{+}^{\mathrm{lr}}$ are positive constants. The large value asymptotics of $X^{\mathrm{lr}}(x)$ ensures that the correlation function decays as $r^{-(d+\sigma)}$ for $r \gg r^{*}$, which is in full agreement with (1.6), and that $r^{*}=(\sigma-2+\eta) \xi \ln \xi$, which coincides with the result of Kayser and Raveché [14]. The property $G(\mathbf{r} ; T \mid d) \simeq J(r) \chi^{2} / \beta$, $r \rightarrow \infty$ 14] is retained too. 
We emphasize, nevertheless, that despite all of the above features, for the moment (5.1) is only a plausible hypothesis the verification of which is still lacking.

At the very end we note that, according to a recent hypothesis [5], the behavior of the bulk two-point correlation function $G(r ; K \mid d, \sigma)$ can be related to that one of the finite-size susceptibility under periodic boundary conditions. In the present article (see Section IV) we checked this hypothesis within the mean spherical model using the results for the finite-size susceptibility derived in [17]. Definitely, one can extend the calculations presented here to models with $\eta \neq 0$ by using renormalization group techniques.

\section{ACKNOWLEDGEMENTS}

Support by DAAD, by DLR under grant number 50 WM 9911 and by NASA under contract number 1201186 is gratefully acknowledged.

The author thanks Prof. V. Dohm for the stimulating discussions, and Prof. J. Brankov, S. Dietrich, J. Rudnick, N. Tonchev, Dr. M. Krech and Dr. E. Korutcheva for the exchange of information and critical reading of the manuscript. The author is also indebted to Prof. P. Russev for drawing his attention to Ref. [35] concerning the properties of the generalized hypergeometric functions.

[1] M. E. Fisher, J. Math. Phys. 5, 944 (1964).

[2] D. Iagolnitzer and B. Souillard, Phys. Rev. A 16, 1700 (1977).

[3] B. M. McCoy and T. T. Wu, The Two-Dimensional Ising Model, (Harvard University Press, Cambridge, Mass., 1973).

[4] M. E. Fisher and R. J. Burford, Phys. Rev. 156, 583 (1967).

[5] X. S. Chen and V. Dohm, Eur. Phys. J. B 15, 283 (2000).

[6] M. E. Fisher, S.K. Ma and B. G. Nikel, Phys. Rev. Lett. 29, 917 (1972).

[7] S. K. Ma, Phys. Rev. A 7, 2172 (1973).

[8] E. Brèzin, J. Zinn-Justin and J. C. Le Guillou, J. Phys. A 9, L219 (1976).

[9] G. S. Joyce, Phys. Rev. 146, 349 (1966).

[10] G. S. Joyce, in Phase Transitions and Critical Phenomena, edited by Domb and Green, v. 2, 375 (Academic, New York, 1972).

[11] B. Widom, J. Chem. Phys. 41, 74 (1964).

[12] J. E. Enderby, T. Gaskell and N. M. March, Proc. Phys. Soc. 85, 217 (1965).

[13] In order to write the above equations in the form given we have taken the number density of the fluid to be $\rho=1$.

[14] R. F. Kayser and H. J. Raveché, Phys. Rev. A 29, 1013 (1984).

[15] G. Flöter and S. Dietrich, Z. Phys. B 97, 213 (1995).

[16] There the reader is also referred to one unpublished, unfortunately, work of S. Dietrich.

[17] D. Dantchev and J. Rudnick, Eur. Phys. J. B 21, 251 (2001).

[18] T. H. Berlin and M. Kac, Phys. Rev. 88, 821 (1952).

[19] H. W. Lewis and G. H. Wannier, Phys. Rev. 88, 682 (1952); 90, 1131 (1953).

[20] J. G. Brankov, D. M. Danchev, N. S. Tonchev, The Theory of Critical Phenomena in Finite-Size Systems Scaling and Quantum Effects, World Scientific, Singapore, 2000.

[21] D. J. Amit, Field Theory, the Renormalization Group, and Critical Phenomena, World Scientific, Singapore, 1984.

[22] X. S. Chen and V. Dohm, in preparation.

[23] C. Domb, The Critical Point, Taylor and Francis, London, 1996.

[24] M. Fisher, in Critical Phenomena, Proc. 51st Enrico Fermi Summer School, Varenna, edited by M. S. Green (Academic Press, New York, 1972).

[25] M. Fisher and M. Barber, Phys. Rev. Lett. 28, 1516 (1972)

[26] M. Barber, in Phase Transitions and Critical Phenomena, v. 8, edited by C. Domb and J. L. Lebowitz (Academic Press, New York, 1983).

[27] V. Privman, in Finite Size Scaling and Numerical Simulation of Statistical Systems, edited by V. Privman (World Scientific, Singapore, 1990).

[28] M.E. Fisher and V. Privman, Commun. Math. Phys. 103, 527 (1986).

[29] X. S. Chen and V. Dohm, Eur. Phys. J. B 10, 687 (1999); 7, 183 (1999).

[30] S. Singh and R. K. Pathria, Phys. Rev. B 40, 9234 (1989). 
[31] J. Brankov and D. Danchev, J. Math. Phys. 32, 9234 (1991).

[32] H. Chamati and N. S. Tonchev, Phys. Rev. E. 63, 026103 (2001).

[33] M. Abramowitz and I. A. Stegun, Handbook of Mathematical Functions, Dover Publications Inc., New York (1970).

[34] I. S. Gradsteyn and I. M. Ryzhik, Table of Integrals, Series, and Products, Academic Press, New York and London, 1973.

[35] Y. L. Luke, Mathematical Functions and their Approximations, Academic Press, New York, 1975.

\section{APPENDIX A: MATHEMATICAL DETAILS}

In this Section we will provide the mathematical details needed to derive Eqs. (3.3), (3.10), (3.11) and (3.12).

Let us start with the case $d=\sigma=3$. Then

$$
G(\mathbf{r} ; K \mid 3,3)=\frac{1}{K} \frac{1}{2 \pi^{2}} \frac{1}{r} \int_{0}^{\infty} f(k) \sin (k r) d k,
$$

where

$$
f(k):=\frac{k}{\xi^{-2}+k^{2}-b k^{3}+c k^{4}} .
$$

It is easy to show that if for a given integer $n>0$ the derivatives $f^{(p)}(0)$ of the function $f$ do exist for $p=0, \cdots, 4 n+2$ and, in addition, $f^{(p)}(\infty)=0, p=0, \cdots, 4 n+2$, then

$$
\int_{0}^{\infty} f(k) \sin (k r) d k=r^{-1} \sum_{p=0}^{n}\left[f^{(4 p)}(0) r^{-4 p}-f^{(4 p+2)}(0) r^{-4 p-2}\right]+O\left(r^{-4 p-5}\right) .
$$

Applying this to (A1) in the limit $r \gg \xi$ one immediately obtains (3.11).

In order to derive Eq. (3.12) let us note that $\left(\xi^{-2}=0\right.$ at $\left.K=K_{c}\right)$

$$
\begin{aligned}
G\left(\mathbf{r} ; K_{c} \mid 3,3\right) & =\frac{1}{K_{c}} \frac{1}{2 \pi^{2}} \frac{1}{r} \int_{0}^{\infty} \frac{1}{1-b k+c k^{2}} \frac{\sin (k r)}{k} d k \\
& =\frac{1}{K_{c}} \frac{1}{2 \pi^{2}} \frac{1}{r}\left[\frac{\pi}{2}+b \int_{0}^{\infty} f_{b}(k) \sin (k r) d k-c \int_{0}^{\infty} f_{c}(k) \sin (k r) d k\right],
\end{aligned}
$$

where

$$
f_{b}(k):=\frac{1}{1-b k+c k^{2}}
$$

and

$$
f_{c}(k):=\frac{k}{1-b k+c k^{2}},
$$

and use has been made of the fact that $\int_{0}^{\infty} \sin (k) / k=\pi / 2$. Applying again (A3) for the evaluation of the integrals in Eq. (A4), we obtain the result given in Eq. (3.12).

The derivation of Eq. (3.10) is a bit more complicated. First, let us note that

$$
\begin{aligned}
G(\mathbf{r} ; K \mid 3,3)= & \frac{K_{c}}{K} G\left(\mathbf{r} ; K_{c} \mid 3,3\right)+\frac{1}{K} \frac{1}{2 \pi^{2}} \frac{1}{r} \int_{0}^{\infty}\left[\frac{k^{2}}{\xi^{-2}+k^{2}-b k^{3}+c k^{4}}-\frac{k^{2}}{k^{2}-b k^{3}+c k^{4}}\right] \frac{\sin (k r)}{k} d k \\
= & \frac{K_{c}}{K} G\left(\mathbf{r} ; K_{c} \mid 3,3\right)-\frac{1}{K} \frac{1}{2 \pi^{2}} \frac{\xi^{-2}}{r} \int_{0}^{\infty} \frac{1}{\left(1-b k+c k^{2}\right)\left(\xi^{-2}+k^{2}-b k^{3}+c k^{4}\right)} \frac{\sin (k r)}{k} d k \\
\simeq & \frac{K_{c}}{K} G\left(\mathbf{r} ; K_{c} \mid 3,3\right)-\frac{1}{K} \frac{1}{2 \pi^{2}} \frac{\xi^{-2}}{r}\left[\int_{0}^{\infty} \frac{1}{\left(1-b k+c k^{2}\right)\left(\xi^{-2}+k^{2}\right)} \frac{\sin (k+}{k} d k\right] \\
& \left.b \int_{0}^{\infty} \frac{k^{3}}{\left(\xi^{-2}+k^{2}\right)^{2}} \frac{\sin (k r)}{k} d k\right]
\end{aligned}
$$




$$
\begin{aligned}
\simeq & \frac{K_{c}}{K} G\left(\mathbf{r} ; K_{c} \mid 3,3\right)-\frac{1}{K} \frac{1}{2 \pi^{2}} \frac{\xi^{-2}}{r}\left[\int_{0}^{\infty} \frac{1}{\xi^{-2}+k^{2}} \frac{\sin (k r)}{k} d k+b \int_{0}^{\infty} \frac{1}{\xi^{-2}+k^{2}} \sin (k r) d k+\right. \\
& \left.b \int_{0}^{\infty} \frac{k^{2}}{\left(\xi^{-2}+k^{2}\right)^{2}} \frac{\sin (k r)}{k} d k\right] \\
= & \frac{K_{c}}{K} G\left(\mathbf{r} ; K_{c} \mid 3,3\right)-\frac{1}{K} \frac{1}{2 \pi^{2}} \frac{\xi^{-2}}{r}\left[\int_{0}^{\infty} \frac{1}{\xi^{-2}+k^{2}} \frac{\sin (k r)}{k} d k+b\left(2+\xi^{-2} \frac{\partial}{\partial \xi^{-2}}\right) \int_{0}^{\infty} \frac{1}{\xi^{-2}+k^{2}} \sin (k r) d k\right] .
\end{aligned}
$$

Above we have already dealt with the large distance asymptotic of $G\left(\mathbf{r} ; K_{c} \mid 3,3\right)$. In order to obtain (3.10) now it only remains to note that 34]

$$
\xi^{-2} \int_{0}^{\infty} \frac{1}{\xi^{-2}+k^{2}} \frac{\sin (k r)}{k} d k=\frac{\pi}{2}\left[1-\exp \left(-\frac{r}{\xi}\right)\right]
$$

and

$$
\int_{0}^{\infty} \frac{\sin (k)}{x^{2}+k^{2}} d k=\frac{1}{2 x}[\exp (-x) \operatorname{Ei}(x)-\exp (x) \operatorname{Ei}(-x)], \operatorname{Re}(x)>0 .
$$

At the end, let us derive the results given in Eq. (3.3). The case $1 \ll r \ll \xi$, i.e. $x \rightarrow 0$, is simple - using the series representations of the modified Bessel functions $I_{a}(x)$ (see, e.g. [33] or [34]) one obtains from Eqs. (2.12)-(2.14) the asymptotics of $X^{\operatorname{lr}}(x)$ for small values of the argument, given in Eq. (3.3). Much more interesting is the case when $r \gg \xi \gg 1$, i.e. when $x \gg 1$. We will present here a derivation of the corresponding asymptotic of $X^{\operatorname{lr}}(x)$ without making use of the large value asymptotic results for the function ${ }_{1} F_{2}$. One can get an impression of the beauty of the proposed way of acting only after taking a look at the results available for the function ${ }_{1} F_{2}$ (see, e.g., [35]). We start by noting that

$$
\begin{aligned}
X^{\operatorname{lr}}(x) & =\int_{0}^{\infty} d t \frac{t^{\sigma+d / 2}}{\left(x+t^{2}\right)^{2}} J_{d / 2-1}(t) \\
& =\left(1+x \frac{\partial}{\partial x}\right) \int_{0}^{\infty} d t \frac{t^{\sigma+d / 2}}{x+t^{2}} J_{d / 2-1}(t) \\
& =\left(1+x \frac{\partial}{\partial x}\right) \int_{0}^{\infty} d z \exp (-z x) \int_{0}^{\infty} d t \exp \left(-z t^{2}\right) t^{\sigma+d / 2-2} J_{d / 2-1}(t) .
\end{aligned}
$$

In order to evaluate the last integral in (A10) one can use the formula 34]

$$
\int_{0}^{\infty} d x x^{\mu} \exp \left(-\alpha x^{2}\right) J_{\nu}(\beta x)=\frac{\beta^{\nu} \Gamma(\nu / 2+\mu / 2+1 / 2)}{2^{\nu+1} \alpha^{(\mu+\nu+1) / 2} \Gamma(\nu+1)}{ }_{1} F_{1}\left(\frac{\nu+\mu+1}{2} ; \nu+1 ;-\frac{\beta^{2}}{4 \alpha}\right) .
$$

With its help one obtains

$$
\int_{0}^{\infty} d t \exp \left(-z t^{2}\right) t^{\sigma+d / 2-2} J_{d / 2-1}(t)=\frac{\Gamma\left(\frac{d+\sigma}{2}-1\right)}{2^{d / 2} \Gamma\left(\frac{d}{2}\right)} z^{1-(d+\sigma) / 2}{ }_{1} F_{1}\left(\frac{d+\sigma}{2}-1 ; \frac{d}{2} ;-\frac{1}{4 z}\right),
$$

i.e.

$$
X^{\operatorname{lr}}(x)=\frac{\Gamma\left(\frac{d+\sigma}{2}-1\right)}{2^{d / 2} \Gamma\left(\frac{d}{2}\right)}\left(1+x \frac{\partial}{\partial x}\right) \int_{0}^{\infty} d z \exp (-z x) z^{1-(d+\sigma) / 2}{ }_{1} F_{1}\left(\frac{d+\sigma}{2}-1 ; \frac{d}{2} ;-\frac{1}{4 z}\right) .
$$

Note now that when $x \gg 1$ the main contribution of the integral in the above expression will stem from small $z$ values. Using the corresponding asymptotic [33]

$$
{ }_{1} F_{1}(a ; b ;-y)=\frac{\Gamma(b)}{\Gamma(b-a)} y^{-a}\left(1+a(a-b+1) y^{-1}+O\left(y^{-2}\right)\right)
$$

of ${ }_{1} F_{1}(a ; b,-y)$ for $y \gg 1$ and performing the integrations, we arrive at the asymptotic of $X^{\mathrm{lr}}(x)$ reported in Eq. (3.3) for the case $x \gg 1$. 